\title{
A Quick Simulation Method for Fading Communications Channels using a Novel Eigenvalue Importance Sampling Technique
}

\author{
Jaunty T. Y. Ho \\ Department of Telecommunications Engineering \\ RSISE, The Australian National University \\ Canberra ACT 0200, Australia \\ e-mail: jaunty.ho@anu.edu.au
}

\author{
Peter J. Smith \\ Department of Electrical and Computer Engineering \\ University of Canterbury \\ Christchurch, New Zealand \\ e-mail: p.smith@elec.canterbury.ac.nz
}

\begin{abstract}
In this paper, we introduce a quick simulation method for fading communications channels using a novel eigenvalue importance sampling technique. Our approach is motivated by the fact that many performance analyses involve metrics which are functions of the eigenvalues of the channel correlation matrix. More specifically in Rayleigh fading we often require the eigenvalues of the Wishart matrix $\mathrm{HH}^{\dagger}$ where $\mathrm{H}$ is the matrix of channel gains. Hence we propose direct simulation of the Wishart eigenvalues rather than simulation of the full channel matrix. If $\mathbf{H}$ is $n_{R} \times n_{T}$ then this idea in itself reduces simulation time since $m=$ $\min \left(n_{R}, n_{T}\right)$ eigenvalues are required rather than the $2 \times n_{R} \times n_{T}$ real Gaussians. However, direct generation of the eigenvalues is complicated. Therefore we introduce a novel eigenvalue importance sampling technique which generates the eigenvalues from a simple biased density which "mimics" the real density. We call our approach Eigenvalue Importance Sampling (EVIS). Secondly, we try to reduce rare event simulation time by using biased eigenvalue densities to encourage the rare event of interest. We denote this approach Rare event Eigenvalue Importance Sampling (REVIS). Both methods are demonstrated via the example of simulating capacity outages and values for a MIMO system. Results show that considerable savings are offered by this novel approach even with simple implementations and small scale systems $(m \leq 4)$.
\end{abstract}

\section{INTRODUCTION}

The analysis of a large number of communication systems over fading channels depends on the channel correlation matrix. An example of great current interest is the analysis of MIMO systems. For a MIMO system there are $n_{T}$ transmit antennas communicating with $n_{R}$ receive antennas and the received signal is,

$$
\mathbf{r}=\mathbf{H} \mathbf{s}+\mathbf{n}
$$

where $\mathbf{r}$ is the $n_{R} \times 1$ received signal vector, $\mathbf{s}$ is the complex $n_{T} \times 1$ transmitted signal vector and $\mathbf{H}$ is an $n_{R} \times n_{T}$ complex channel gain matrix. The AWGN vector $\mathbf{n}$ consists of $n_{R}$ independent noise components of modulus variance normalised to one. The capacity of such a system is now very well known $[1,2]$ and assuming equal power sources is given by

$$
C=\log _{2}\left[\operatorname{det}\left(\mathbf{I}_{n_{R}}+\frac{P}{n_{T}} \mathbf{H} \mathbf{H}^{\dagger}\right)\right]
$$

where $\mathbf{I}_{n_{R}}$ is the $n_{R} \times n_{R}$ identity matrix and $P$ is the total transmit power. It is important to note that the capacity has the alternative representation [2]

$$
C=\sum_{i=1}^{m} \log _{2}\left(1+\frac{P}{n_{T}} \lambda_{i}\right)
$$

where $m=\min \left(n_{R}, n_{T}\right)$ and $\lambda_{1}, \lambda_{2}, \cdots, \lambda_{m}$ are the eigenvalues of $\mathbf{W}$ where

$$
\mathbf{W}=\left\{\begin{array}{ll}
\mathbf{H H}^{\dagger} & n_{R} \leq n_{T} \\
\mathbf{H}^{\dagger} \mathbf{H} & n_{T}<n_{R}
\end{array} .\right.
$$

Hence the capacity of a MIMO system is solely a function of $m$ real eigenvalues. This is the starting point for our approach. For large values of $n_{R}$ and $n_{T}$ a straight forward simulation of (2) involves $n_{R} \times n_{T}$ complex random variables. Direct simulation of (3) on the other hand requires only $m$ real random variables. The MIMO example, however, is only one possibility. The work in this paper can be applied to many communications problems. For example, the performance of diversity combiners such as ZF or MMSE in SIMO or MIMO systems are functions of the SINR, which in turn is a function of the eigenvalues of the channel correlation matrix [3,4]. The same is also true for certain multiuser receivers [5]. We focus on the independent Rayleigh fading scenario, although the approach can be extended to other cases.

In this paper we focus on the direct simulation of eigenvalues rather than the simulation of the full channel matrix. Our work can be further split into two parts. Firstly we seek to reduce simulation time by direct simulation of the $m$ eigenvalues rather than the $2 \times n_{R} \times n_{T}$ fading variables. We call our approach Eigenvalue Importance Sampling (EVIS). Secondly, and more traditionally, we try to reduce rare event simulation time by using biased eigenvalue densities to encourage the rare events of interest. We denote this approach Rare event Eigenvalue Importance Sampling (REVIS). The main ideas of these techniques and the mathematics behind the methodology will be included in the paper. We will implement these ideas for the MIMO capacity example. Some results will also be given to show the savings offered by this novel IS approach. 


\section{EigenVAlue Importance SAMPLing (EVIS)}

\section{A. EVIS derivation}

Our EVIS approach is different from most applications of Importance Sampling (IS) in communications [6] which seek to reduce the simulation time of rare events. Instead, EVIS is used to reduce the complexity of random variable generation from a complicated density. As shown in [2], the joint distribution of the eigenvalues of $W$ is

$$
\begin{gathered}
f\left(\lambda_{1} \ldots \lambda_{m}\right)=\frac{2^{-m n} \pi^{m(m-1)}}{m ! \tilde{\Gamma}_{m}(n) \tilde{\Gamma}_{m}(m)} \exp \left(-\frac{1}{2} \sum_{i=1}^{m} \lambda_{i}\right) \\
\times \prod_{i=1}^{m} \lambda_{i}^{n-m} \prod_{i<j}\left(\lambda_{i}-\lambda_{j}\right)^{2} \\
\tilde{\Gamma}_{m}(a)=\pi^{m(m-1) / 2} \prod_{i=1}^{m} \Gamma(a-i+1) \quad \lambda_{1} \leq \lambda_{2} \leq \cdots \leq \lambda_{m}
\end{gathered}
$$

where $\lambda_{1}, \lambda_{2}, \cdots, \lambda_{m}$ are the $m$ eigenvalues of $\mathbf{W}$ in (4), $n=\max \left(n_{R}, n_{T}\right)$ and $\Gamma(\cdot)$ is the gamma function. Generating random variables from this density is not an attractive option. Therefore, we employ IS and generate from a biased but trivial eigenvalue density which attempts to mimic the awkward true density (5). For example suppose we are interested in estimating the probability, $\mathrm{p}$, that the capacity exceeds a threshold $T$. Here we define the indicator function $D(C)$ such that $D(C)=0$ if $C \leq T$ and $D(C)=1$ if $C>T$. With this notation the IS estimator, $\hat{p}_{I S}$, is given by

$$
\hat{p}_{I S}=\frac{1}{n} \sum_{i=1}^{n} D\left(C\left(\lambda_{1}^{(i)}, \cdots, \lambda_{m}^{(i)}\right)\right) w\left(\lambda_{1}^{(i)}, \cdots, \lambda_{m}^{(i)}\right)
$$

where $w\left(\lambda_{1}, \cdots, \lambda_{m}\right)=\frac{f\left(\lambda_{1}, \cdots, \lambda_{m}\right)}{f^{*}\left(\lambda_{1}, \cdots, \lambda_{m}\right)}, f(\cdot)$ is the true density and $f^{*}(\cdot)$ is the biased density. Note that the notation $C\left(\lambda_{1}, \cdots, \lambda_{m}\right)$ simply represents the fact that the capacity is a function of the $m$ eigenvalues. In other words, we generate the eigenvalues from the trivial biased density function $f^{*}(\cdot)$ and then scale the results by the weight factor $w(\cdot)$. The expected value of $\hat{p}_{I S}$ is indeed equal to the true probability. This is well known [6] but is proven below for completeness,

$$
\begin{aligned}
E_{*}\left(\hat{p}_{I S}\right) & =\frac{1}{n} \sum_{i=1}^{n} \int D\left(C\left(\boldsymbol{\lambda}^{(i)}\right)\right) w\left(\boldsymbol{\lambda}^{(i)}\right) f^{*}\left(\boldsymbol{\lambda}^{(i)}\right) \boldsymbol{d} \boldsymbol{\lambda}^{(i)} \\
& =\quad \int D\left(C\left(\boldsymbol{\lambda}^{(i)}\right)\right) f\left(\boldsymbol{\lambda}^{(i)}\right) \boldsymbol{d} \boldsymbol{\lambda}^{(i)}=p,
\end{aligned}
$$

where $\boldsymbol{\lambda}^{(i)}=\left[\lambda_{1}^{(i)}, \cdots, \lambda_{m}^{(i)}\right]$. Note that the * subscript in (7) refers to expectation over the biased density.

\section{B. The choice of biased density $f^{*}(\cdot)$}

To illustrate the EVIS idea here, we consider a $2 \rightarrow 2$ MIMO system and plot the true density $f\left(\lambda_{1}, \lambda_{2}\right), \lambda_{1} \leq \lambda_{2}$ in Fig. 1 . Our aim in EVIS is to find a biased density $f^{*}(\cdot)$ that generates random eigenvalues easily and has a similar shape to the true density $f(\cdot)$. We proceed as follows. Let $\lambda_{1} \leqslant \lambda_{2} \leqslant \cdots \leqslant$ $\lambda_{m}$ be the ordered eigenvalues of $\mathbf{W}$ and let $G(r, \theta)$ denote a

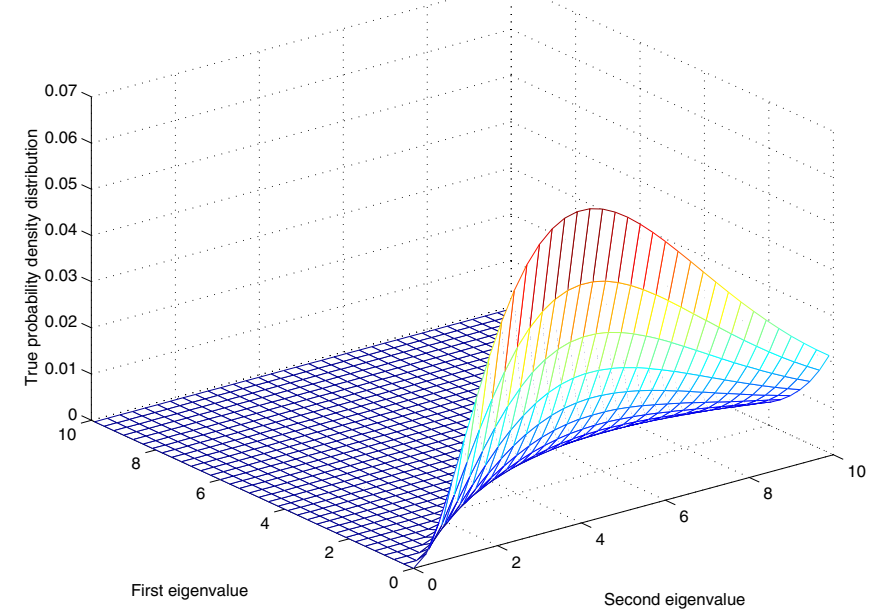

Fig. 1. This figure shows the true density distribution $f\left(\lambda_{1}, \lambda_{2}\right)$ where $\lambda_{1} \leq$ $\lambda_{2}$. We propose a technique (EVIS) to generate eigenvalues from a biased but trivial density distribution $f^{*}(\cdot)$. We aim to choose $f^{*}(\cdot)$ so that it is simple to generate eigenvalues and has a similar shape to $f(\cdot)$.

gamma distribution with shape parameter $r$ and scale parameter $\theta$. The density for such a distribution is given by

$$
\frac{1}{\theta^{r} \Gamma(r)} \lambda^{r-1} \exp \left(-\frac{\lambda}{\theta}\right) .
$$

We generate the biased eigenvalues as follows:

$$
\lambda_{k}=\sum_{i=1}^{k} X_{i} \quad, k=1,2, \cdots, m
$$

where $X_{1}, X_{2}, X_{3}, \cdots, X_{m}$ are mutually independent and $X_{k} \backsim G\left(r_{k}, \theta_{k}\right)$. Our use of gamma variables in (9) is driven by the following observations:

- For SIMO and MISO systems, (5) can be simplified to

$$
f\left(\lambda_{1}\right)=2^{-n} \lambda_{1}^{n-1}[(n-1) !]^{-1} e^{-\lambda_{1} / 2}
$$

which is the density of a $G(n, 2)$ variable. Also for the $2 \rightarrow$ 2 MIMO system some simple calculations show that $\lambda_{1} \backsim$ $G(1,1)$ and $\lambda_{2} \backsim \lambda_{1}+G(3,2)$. Therefore (9) is exact for SIMO, MISO and $2 \rightarrow 2$ MIMO systems.

- The marginal distributions of $\lambda_{k}, k=1,2, \cdots, m$ give excellent goodness-of-fit results when simulations are compared to gamma distributions. Since sums of gamma variables are closely approximated by a single gamma variable this also supports the use of (9). Similarly, simulations of the eigenvalue spacings, $\lambda_{k}-\lambda_{k-1}, k \geq 2$, also show good fit to gamma distributions.

- Foschini's capacity lower bound [1] uses chi-squared random variables (special cases of gamma variables) to replace the eigenvalues. In certain situations the bound is quite tight, again suggesting the use of gamma variables.

Once we have chosen the biasing method, (9), the next step is parameter choice. Here we are aided by large sample results. We know that when $n_{R}$ and $n_{T}$ are large, the limiting eigenvalue distribution of $\mathbf{H H}^{\dagger} / n_{T}$ is given by [7] as 


$$
\begin{aligned}
& f(\lambda)=\frac{(\sqrt{\lambda-a(\rho)})(\sqrt{b(\rho)-\lambda})}{2 \pi \rho \lambda} \quad a(\rho)<\lambda<b(\rho) \\
& =0 \quad 0 \quad \text { otherwise }
\end{aligned}
$$

where $\rho=r / t, a(\rho)=1+\rho-2 \sqrt{\rho}$ and $b(\rho)=1+\rho+$ $2 \sqrt{\rho}$. Note that (11) refers to the density of a randomly selected eigenvalue. Other results [8] show how quickly this distribution becomes accurate. For example, when $n_{R}=n_{T}=10$, the ordered eigenvalues of $\mathbf{H H}^{*} / n_{T}$ tend to be located in a specific pattern between $0<\lambda<4$ as shown in Fig. 2. It can be seen that the eigenvalues have a surprisingly well-behaved structure and this helps in proposing parameters for the biased densities.

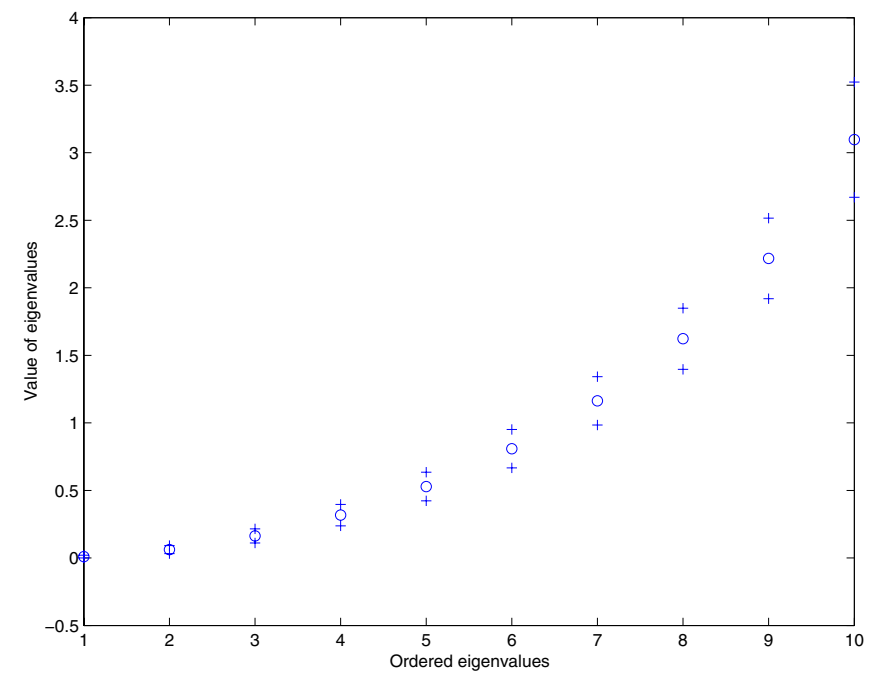

Fig. 2. This figure shows the mean value "o" of the eigenvalues $\lambda_{1} \leq \lambda_{2} \leq$ $\cdots \leq \lambda_{10}$, with $+/$ - one standard derivation " + ". The eigenvalues have a surprisingly well-behaved structure and tend to lie between 0 and 4 . This aids parameter selection for the biased distributions.

\section{Choice of gamma variable parameters}

In the previous section, we mentioned the reasons for generating the biased eigenvalues by sums of gamma variables as in (8). In this section we propose a method to select the parameters $r_{k}$ and $\theta_{k}$, where $1 \leq k \leq m$. One sensible way to proceed is to choose the parameters such that the expected value and variance of $\lambda_{k}$ are correct. Hence we must satisfy

$$
\begin{gathered}
E\left\{\lambda_{k}\right\}=E\left\{\sum_{i=1}^{k} X_{i}\right\}=\sum_{i=1}^{k} E\left\{X_{i}\right\}=\sum_{i=1}^{k} r_{i} \theta_{i} \\
\operatorname{var}\left\{\lambda_{k}\right\}=\operatorname{var}\left\{\sum_{i=1}^{k} X_{i}\right\}=\sum_{i=1}^{k} \operatorname{var}\left\{X_{i}\right\}=\sum_{i=1}^{k} r_{i} \theta_{i}^{2}
\end{gathered}
$$

for $1 \leq k \leq m$. Once we have $E\left\{\lambda_{k}\right\}$ and $\operatorname{var}\left\{\lambda_{k}\right\}$, we can obtain the gamma parameters $r_{k}$ and $\theta_{k}$ using (12) and (13), such that

$$
\begin{array}{rlrl}
\theta_{1} & =\frac{\operatorname{var}\left\{\lambda_{1}\right\}}{E\left\{\lambda_{1}\right\}} & r_{1} & =\frac{E\left\{\lambda_{1}\right\}}{\theta_{1}} \\
\theta_{2} & =\frac{\operatorname{var}\left\{\lambda_{2}\right\}-r_{1} \theta_{1}^{2}}{E\left\{\lambda_{2}\right\}-r_{1} \theta_{1}} & r_{2} & =\frac{E\left\{\lambda_{2}\right\}-r_{1} \theta_{1}}{\theta_{2}} \\
\theta_{3} & =\frac{\operatorname{var}\left\{\lambda_{3}\right\}-r_{2} \theta_{2}^{2}-r_{1} \theta_{1}^{2}}{E\left\{\lambda_{3}\right\}-r_{2} \theta_{2}-r_{1} \theta_{1}} & r_{3} & =\frac{E\left\{\lambda_{3}\right\}-r_{2} \theta_{2}-r_{1} \theta_{1}}{\theta_{3}} \\
& \vdots & \vdots
\end{array}
$$

Therefore, we only need to find the expected value and variance of $\lambda_{1}, \lambda_{2}, \cdots, \lambda_{m}$ to define the gamma parameters $\theta_{1}, \theta_{2}, \cdots, \theta_{m}$ and $r_{1}, r_{2}, \cdots, r_{m}$. Remember that (11) gives the limiting eigenvalue distribution of $\mathbf{H H}^{*} / n_{T}$. Let us now consider the case that $n_{R}=n_{T}$ (a similar approach can be applied to other combinations of $n_{R}$ and $n_{T}$ ), then (11) becomes

$$
f(\lambda)=\frac{1}{\pi} \sqrt{\frac{1}{\lambda}-\frac{1}{4}} \quad 0<\lambda<4 .
$$

Now let us integrate (15), and the cumulative distribution function $F(\lambda)$ is

$$
F(\lambda)=\frac{1}{\pi}\left\{\frac{\pi}{2}+\sqrt{\frac{4 \lambda-\lambda^{2}}{4}}-\sin ^{-1}\left(1-\frac{\lambda}{2}\right)\right\} \quad 0<\lambda<4 .
$$

Fig. 3 shows the cumulative distribution function of the eigenvalues of $\mathbf{H H}^{*} / n_{T} . F(\lambda)$ is very smooth and well-behaved.

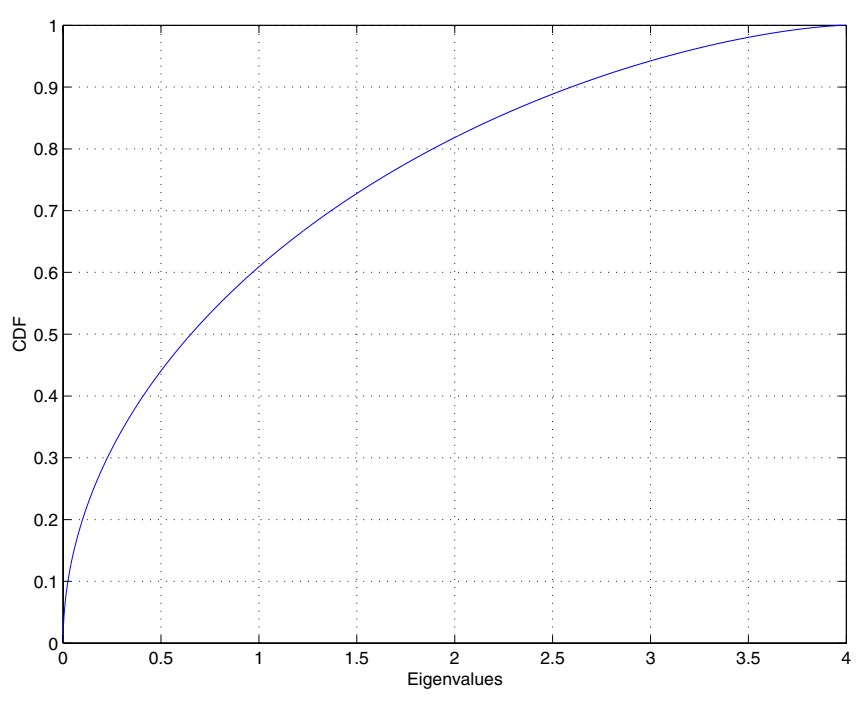

Fig. 3. The cumulative distribution function of the eigenvalues of $\mathbf{H H}^{*} / n_{T}$. $F(\lambda)$ is very smooth and well-behaved.

Now we apply the approximate result in order statistics [9]

$$
E\left(X_{i}\right) \approx F^{-1}\left(\frac{i}{m+1}\right)
$$


for a sample of size $m$ where $X_{1}, X_{2}, \cdots, X_{m}$ are samples from $F$ and $X_{1} \leq X_{2} \leq \cdots \leq X_{m}$. Therefore, we can approximate the expected value of the the eigenvalues by

$$
E\left(\lambda_{k}\right) \approx F^{-1}\left(\frac{k}{m+1}\right) .
$$

Note that this is a plausibility argument only. The eigenvalues are not random samples from the density in (15). Nevertheless applying the approximation in (17) is extremely accurate, as can be seen from reading off the approximation from Fig. 3 and comparing the result to Fig. 2. Similarly, the variance of the eigenvalues is estimated by

$$
\operatorname{var}\left(\lambda_{k}\right) \approx \frac{\left(\frac{k}{m+1}\right)\left(1-\frac{k}{m+1}\right)}{m+2}\left\{\left.\frac{d}{d x} F^{-1}(x)\right|_{x=\frac{k}{m+1}}\right\}^{2} .
$$

Note that $\frac{d}{d x} F^{-1}(x)=\frac{1}{f\left(F^{-1}(x)\right.}$, therefore, (19) becomes

$$
\operatorname{var}\left(\lambda_{k}\right) \approx \frac{\left(\frac{k}{m+1}\right)\left(1-\frac{k}{m+1}\right)}{m+2}\left\{\frac{1}{f\left(F^{-1}\left(\frac{k}{m+1}\right)\right)}\right\}^{2} .
$$

Now we only need $f(\lambda)$ and $F(\lambda)$ to approximate the expected value and variance of eigenvalues. The inverse of $F, F^{-1}(x)$, can be easily obtained by the use of Fig. 3 . The procedure for choosing gamma parameters is summarized as follows:

1) Use (18) and (20) to estimate the expected value $E\left(\lambda_{k}\right)$ and variance $\operatorname{var}\left(\lambda_{k}\right)$ of the eigenvalue $\lambda_{k}$, where $k=$ $\{1,2, \cdots, m\}$.

2) Use (14) to select the gamma parameters, $r_{k}$ and $\theta_{k}$.

\section{Results}

In this section we evaluate the EVIS technique by computing the runtime savings achieved while estimating the mean capacity of a $4 \rightarrow 4$ MIMO system. The runtime saving formula we use is as follows,

$$
\text { saving }=\frac{\operatorname{var}_{M C}}{\operatorname{var}_{I S}} \times \frac{\operatorname{comp}_{M C}}{\operatorname{comp}_{I S}}
$$

where var and comp are the sample variance of the simulated capacity values and the computational time for the specific simulation technique, respectively. Running the simulation 10, 000 times in MATLAB gave a run time saving of 2.95. Fig. 4 and 5 show histograms of the simulated capacity values using the methods described in (2) and (3). Note that this is for a $4 \rightarrow 4$ MIMO system; larger run time saving can be obtained for larger systems. Given that $W$ contains 16 complex Gaussians and our method generates 4 eigenvalues it is unlikely that savings of more than 4 are available with simple techniques. Hence we seem to be achieving reasonable performance.

\section{Rare Event Eigenvalue Importance Sampling (REVIS)}

In this section, we want to reduce the simulation time of the rare event of interest. Unlike the previous section where the biased distribution $f^{*}(\cdot)$ is chosen to mimic the true distribution

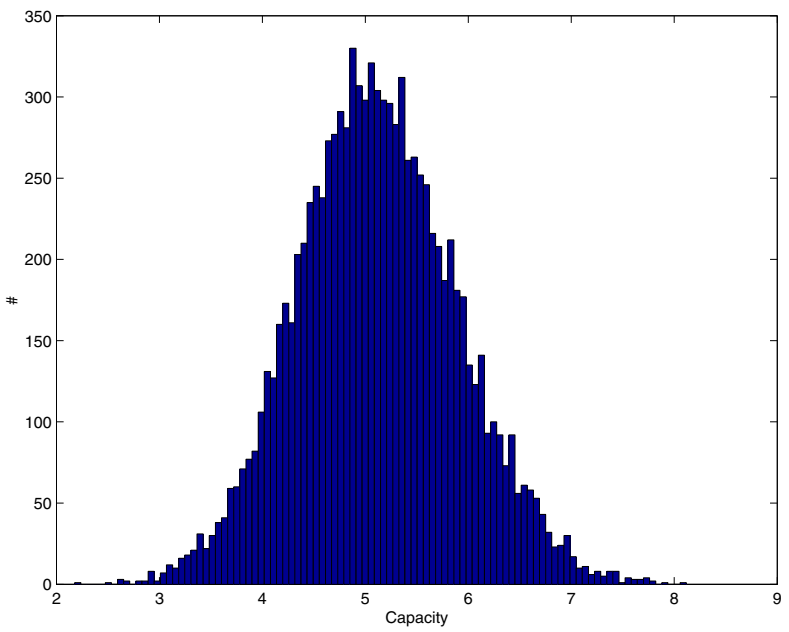

Fig. 4. The capacity histogram for a $4 \rightarrow 4$ MIMO system using Monte Carlo Method with 10,000 simulations. The variance $\operatorname{var}_{M C}$ and the computational time comp $M_{C}$ are 0.607 and 7.110 , respectively.

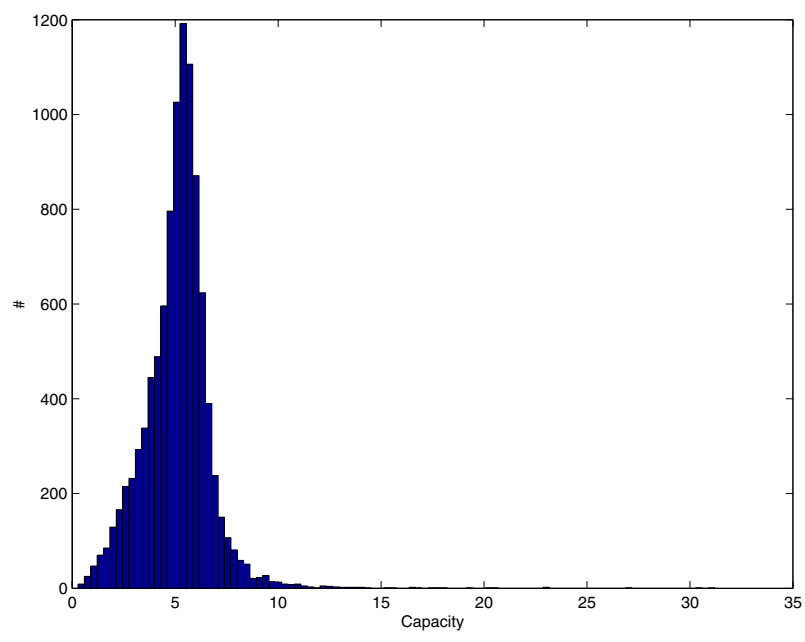

Fig. 5. The capacity histogram for a $4 \rightarrow 4$ MIMO system using EVIS method with 10,000 simulations. The variance $\operatorname{var}_{I S}$ and the computational time comp $I S$ are 2.211 and 0.661 , respectively. This gives a 2.95 run time saving compared to the MC method (Fig.4).

$f(\cdot)$, here we want to have a biased distribution that encourages the rare event to happen. For example, in a MIMO system, we may want to know the probability that the capacity is less than one fifth of the expected value, that is, $\operatorname{Prob}\left(C<\frac{E\{C\}}{5}\right)$. This may require a large number of straight forward Monte Carlo (MC) simulations.

The theory behind REVIS is very similar to EVIS, as described in the previous section. Therefore, we do not intend to reproduce it here. The only difference is that the biased gamma parameters are chosen, not to mimic the true density but to encourage the rare events. We have developed a mean translation method [6] where the biased distribution has its mean values shifted so that the most likely eigenvalues give rise to capacity values on the boundary of the region of interest. However this 
is beyond the scope of this paper.

\section{A. Results}

To illustrate the usefulness of REVIS, we consider a $2 \rightarrow 2$ MIMO system. It is almost the simplest MIMO system, yet despite this REVIS provides large simulation time savings. Based on the observations of the previous section, it is still a good idea to generate the eigenvalue spacings as gamma variables. However, this time we choose a biased distribution to encourage small capacity values. We chose $\lambda_{1}$ and $\lambda_{2}$ to be independent $\mathrm{G}(1,0.5)$ variables. The Monte Carlo capacity histogram and the biased capacity histogram for 100,000 and 500 simulations are shown in Fig. 6 and 7, respectively.

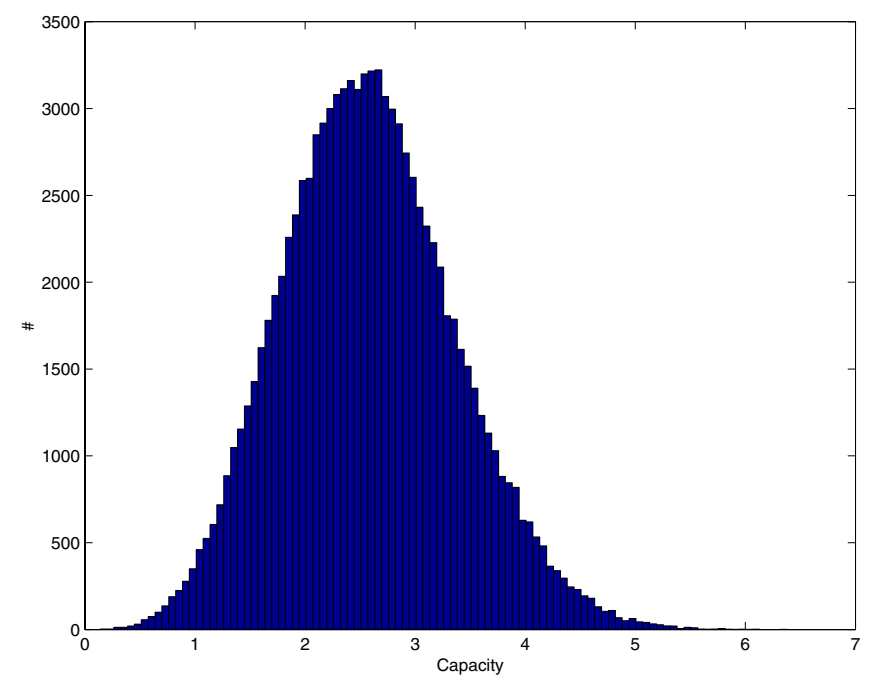

Fig. 6. The capacity histogram for a $2 \rightarrow 2$ MIMO system with 100,000 simulations.

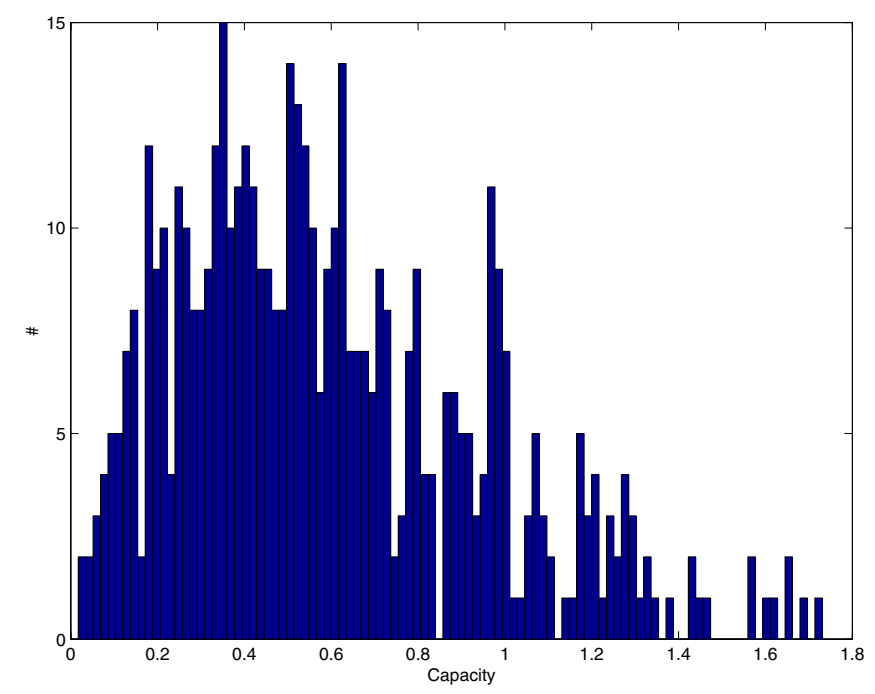

Fig. 7. The biased capacity histogram for a $2 \rightarrow 2$ MIMO system with 500 simulations. Note that we encourage small values for the capacity.

By this simple use of the REVIS approach, we show that 500 simulations is sufficient to determine the desired probabil- ity (see Fig. 8), while the straight forward MC approach was inaccurate even with 100,000 simulations.

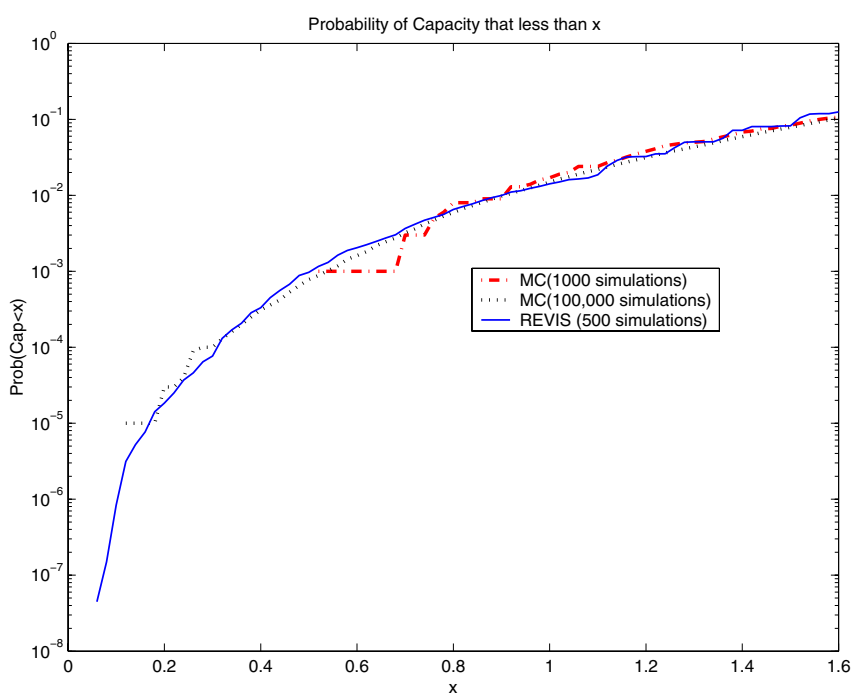

Fig. 8. This figure shows the probability of capacity outage, ie, $P(C<x)$, using a straight forward MC and REVIS approach. Note that the MC approach is inaccurate when $x<0.71$ for 1000 simulations and when $x<0.18$ for 100,000 simulations. The REVIS approach is accurate over the whole range with only 500 simulations.

\section{CONCLUSIONS}

In this paper, we have introduced a new implementation of Importance Sampling in communications systems based on the direct simulation of eigenvalues. We have developed two simple techniques (EVIS and REVIS) and demonstrated their ability to speed up simulation time. A methodology for parameter selection in the EVIS case was given. For the REVIS case a similar method based on traditional IS ideas of mean translation has been developed. This development is more lengthy and so we simply demonstrated the potential gains of a given REVIS approach here.

\section{REFERENCES}

[1] G.J. Foschini and M.J. Gans, "On limits of wireless communication in a fading environment when using multiple antennas," Wireless Personal Communications, Vol.6, No.3, p.311-335, March 1998.

[2] E. Telatar, Capacity of multi-antenna Gaussian channels, Technical Report \# BL0112170-950615-07TM, AT \& T Bell Laboratories, 1995.

[3] J. H. Winters, "Optimum combining in digital mobile radio with co-channel interference," IEEE J Select Areas Commun, Vol. 2, pp. 528-539, July 1984.

[4] H. Gao, P.J. Smith and M.V. Clark, "Theoretical reliability of linear diversity combining in Rayleigh fading, additive interference channels," IEEE Transactions on Communications, vol.46, No.5, pp. 666-672, 1998.

[5] D.N.C. Tse and O. Zeitouni, "Linear multiuser receivers in random environments," IEEE Transactions on Information Theory, Vol. 46 Issue:1, p.171-188, Jan. 2000.

[6] P.J. Smith, M. Shafi, and H. Gao, "Quick simulation: a review of importance sampling techniques in communications systems," IEEE Jour. Selected Areas Commun., JSAC-15, pp.597-613, May 1997.

[7] P.B. Rapajic and D. Popesu, "Information capacity of a random signature multiple-input multiple-output channel," IEEE Transactions on Communications, Vol.48, No.8, p.1245-1248, August 2000.

[8] P.J. Smith and M. Shafi, "On a Gaussian approximation to the capacity of wireless MIMO systems," to appear: IEEE ICC conference, New York, 2002.

[9] H.A. David, Order Statistics, Wiley, New York, 1981. 